\title{
Effect of Astragalus Polysaccharide on the Expression of VEGF and EGFR in Mice with Lewis Transplantable Lung Cancer
}

\author{
Lichun Zhao, Yute Zhong, Jie Liang, Hongwei Gao and Nong Tang
}

\begin{abstract}
The aim of this study was to determine the effect of Astragalus polysaccharide (APS) on the expression of vascular endothelial growth factor (VEGF) and epidermal growth factor receptor (EGFR) in mice with Lewis transplantable lung cancer. It was an experimental study carried out from June 2017 to January 2018 at the College of Pharmacy, Guangxi University of Chinese Medicine, Nanning, China. Forty male SPF-level C57BL/6J mice were selected and inoculated with Lewis lung cancer cell suspension in the right axilla of the mice to establish a lung-cancer mouse model. The mice were randomly divided into a model control group and APS groups with high, middle and low dosages, respectively, 10 in each group. After inoculation of Lewis tumor cell suspension for 2 days, mice in the model control group were injected intraperitoneally with $50 \mathrm{~mL} / \mathrm{kg}$ of $0.9 \%$ sodium chloride solution, whereas, mice in the APS groups with high, middle and low dosages were intraperitoneally injected with APS at $100,50,25 \mathrm{mg} / \mathrm{kg}$, respectively. The research results showed that APS can effectively inhibit the growth and metastasis of Lewis lung cancer in mice, improve immune organ function, inhibit the protein expression of VEGF and EGFR in tumor tissues, and have a concentration-effect relationships.
\end{abstract}

Key Words: Astragalus polysaccharide (APS), Lewis lung cancer, VEGF, EGFR, Immune organ.

Lung cancer is a high-incidence malignancy. At present, the treatment methods for lung cancer include surgical operation, chemotherapy, traditional treatment, and molecular targeted therapy, etc. ${ }^{1}$ At the early stage, lung cancer is mainly treated by surgery. But about $80 \%$ of patients are diagnosed at the middle or late stage, requiring chemotherapy. However, chemotherapy can inhibit the bone marrow hematopoietic system, and cause concurrent infection and even death. Radiotherapy is not much effective for patients with advanced lung cancer. Astragalus polysaccharide (APS) is a water soluble sugar extracted from Astragalus root or leguminous plants. It has been found to have important immunomodulatory, anti-tumor, antioxidative and other extensive pharmacological effects. ${ }^{2}$ However, the mechanism is not yet clear.

Vascular endothelial growth factor (VEGF) is a proangiogenic factor with the highest specificity currently, which is closely related to the growth and metastasis of tumor cells. Epidermal growth factor receptor (EGFR) is an important epidermal cell growth factor actively involved in tumor growth. Relevant data show that the abnormal expressions of VEGF and EGFR protein play an important role in the development of lung cancer. ${ }^{3}$ The purpose of this study was to analyze the effect of

College of Pharmacy, Guangxi University of Chinese Medicine, Nanning, 530200, China

Correspondence: Dr. Nong Tang, College of Pharmacy, Guangxi University of Chinese Medicine, Nanning, 530200, China

E-mail:bfvbpm56@163.com

Received: June 01, 2018; Accepted: October 13, 2018
Astragalus polysaccharide on the expression of VEGF and EGFR in Lewis transplantable lung cancer in mice, in order to further clarify the mechanism of anti-lung cancer effect of Astragalus polysaccharide.

This study was conducted at the College of Pharmacy, Guangxi University of Chinese Medicine, Nanning, China, from June 2017 to January 2018, after approval from the Committee on Animal Research and Ethics. A total of 40 male SPF C57BL/6J mice were selected as subjects with a body mass of $18-22 \mathrm{~g}$, and a mean body weight of $19.0 \pm 2.0 \mathrm{~g}$. The Lewis tumor-bearing mice that were inoculated with Lewis lung cancer tumors for about 14 days were sacrificed by cervical dislocation and placed in $75 \%$ alcohol to be soaked for 10 mins. The tumor body was isolated on a clean bench and tumor tissues with good growth and no necrosis were obtained. After washing with physiological sodium chloride solution, they were cut and smashed. Next, sodium chloride physiological solution was added to be grinded. Then, they were filtered through a 300-mesh nylon net to generate single-cell suspension, and the cell concentration was adjusted to $1 \times 10^{7}$ cells $/ \mathrm{mL}$ with sodium chloride physiological solution; tryan blue excluding test showed over $95 \%$ cell viability. 4

A lung-cancer Lewis tumor cell suspension of $0.2 \mathrm{~mL}$ was inoculated into the right axilla of $20 \mathrm{C} 57 \mathrm{BL} / 6 \mathrm{~J}$ mice to construct a mouse model of Lewis lung cancer. After modeling, the mice were randomly divided into a control group and an Astragalus polysaccharide group, 10 in each group. Two days after the inoculation of Lewis tumor cell suspension, each group of mice was administered intraperitoneally, namely, the control group was given $50 \mathrm{~mL} / \mathrm{kg}$ of $0.9 \%$ sodium chloride solution once a day for 3 weeks; and APS groups with high, 
Table I: Comparison of relevant indicators in each group.

\begin{tabular}{|c|c|c|c|c|c|}
\hline Parameter & $\begin{array}{l}\text { Model control group } \\
\qquad n=10\end{array}$ & $\begin{array}{c}\text { APS high dose group } \\
(100 \mathrm{mg} / \mathrm{kg}) \\
\mathrm{n}=10\end{array}$ & $\begin{array}{c}\text { APS middle dose group } \\
(50 \mathrm{mg} / \mathrm{kg}) \\
\mathrm{n}=10\end{array}$ & $\begin{array}{c}\text { APS low dose group } \\
(25 \mathrm{mg} / \mathrm{kg}) \\
\mathrm{n}=10\end{array}$ & p-value \\
\hline Number of metastatic nodules $n=10$ & $10.25 \pm 1.98$ & $4.43 \pm 0.37$ & $6.68 \pm 0.96$ & $8.37 \pm 1.08$ & $<0.001$ \\
\hline Wet weight of transplantation tumors $(\mathrm{g}) \mathrm{n}=10$ & $7.27 \pm 1.95$ & $3.85 \pm 0.64$ & $5.24 \pm 1.18$ & $6.16 \pm 1.02$ & $<0.001$ \\
\hline Spleen weight $(\mathrm{g}) \mathrm{n}=10$ & $0.22 \pm 0.04$ & $0.55 \pm 0.09$ & $0.38 \pm 0.06$ & $0.30 \pm 0.05$ & $<0.001$ \\
\hline Spleen index $\left(\times 10^{-3}\right) n=10$ & $11.58 \pm 1.09$ & $28.95 \pm 1.86$ & $20.42 \pm 1.32$ & $15.79 \pm 1.20$ & $<0.001$ \\
\hline Thymus weight $(\mathrm{g}) \mathrm{n}=10$ & $0.20 \pm 0.02$ & $0.66 \pm 0.08$ & $0.43 \pm 0.05$ & $0.35 \pm 0.03$ & $<0.001$ \\
\hline Thymus index $\left(\times 10^{-3}\right)$ & $10.53 \pm 0.81$ & $34.74 \pm 2.02$ & $22.14 \pm 1.03$ & $17.37 \pm 0.85$ & $<0.001$ \\
\hline VEGF/ $\beta$-actin $n=10$ & $0.82 \pm 0.18$ & $0.31 \pm 0.04$ & $0.43 \pm 0.06$ & $0.55 \pm 0.09$ & $<0.001$ \\
\hline EGFR/ $\beta$-actin $n=10$ & $0.84 \pm 0.23$ & $0.35 \pm 0.06$ & $0.47 \pm 0.16$ & $0.56 \pm 0.07$ & $<0.001$ \\
\hline
\end{tabular}

middle and low dosages were injected intraperitoneally with APS (provided by College of Pharmacy, Guangxi University of Chinese Medicine) of $100,50,25 \mathrm{mg} / \mathrm{kg}$, respectively, once daily for 3 weeks. The two groups of mice were fed conventionally during the administration period. After 3 weeks of continuous administration, the mice were sacrificed, the relevant tumor tissues were collected for standby application, and the organs such as the thymus and spleen of the mice were removed.

After the mice were sacrificed, their tumor tissues were peeled off. The histology of these tissues was observed, the number of metastatic nodules on the lung surface was counted, and the wet weight of the transplantation tumor was measured. The immune organ index of spleen and thymus was compared between the two groups. Calculation formula: spleen index = spleen weight $/$ weight, and thymus index = thymus weight/weight. Western blot was used to detect the expression of VEGF and EGFR protein in tumor tissue of each group. 5 The appropriate amount of tumor tissue was collected, cell lysis buffer was instilled for lysis, and centrifuged to keep supernatant, and Western blot was performed. After protein quantification, polyacrylamide gel electrophoresis (SDSPAGE) was performed. Rabbit anti-mouse VEGF, EGFR polyclonal primary antibodies (working concentration $1: 1000)$ and goat anti-rabbit IgG second antibodies remarked with horseradish peroxidase (working concentration 1:2000) were added, respectively. Chemiluminescence was used for developing. B-actin was served as an internal reference.

Collected data were analysed using one-way ANOVA by Statistical Package for Social Sciences (SPSS) version 25. Mean and standard deviations were calculated for different quantitative variables. P-value of less than 0.05 was considered significant.

The tumor was round or lobulated. There was no necrotic area of tumor tissue in model control group. APS groups with high, middle and low dosages respectively, showed different necrotic areas.

The tumor volume of the model control group was the largest and the vascular growth was the most abundant, while the tumor volume of APS high dose group was the smallest and less vascular growth. Number of metastatic nodules and wet weight of transplantation tumors in the
APS groups with high, middle and low dosages respectively, were lower than those in the model control group (all $p<0.001$, Table I), in a dose-dependent manner. Spleen weight, thymus weight, spleen index and thymus index in the APS groups with high, middle and low dosages, respectively, were significantly greater than those in the model control group (all $p<0.001$, Table I), in a dose-dependent manner. Relative expression levels of VEGF and EGFR protein in the tumor tissue of the APS groups with high, middle and low dosages, respectively, were lower than those in the model control group (all $\mathrm{p}<0.001$, Table I).

Astragalus polysaccharide is a main active ingredient of Astragalus membranaceus. Its anti-tumor effect can be exerted by activating the NK cells and macrophages to promote the secretion of cytokines, activate lymphocytes, promote the synthesis of dendritic cells, and enhance the immunity of red blood cells and other immune regulatory pathways. This study showed that the number of metastatic nodules and the wet weight of transplantation tumors of mice in the APS groups were lower than those in the model control group. It indicates that APS can inhibit the growth and metastasis of transplantation tumors of Lewis lung cancer in mice. This conclusion is basically consistent with the previous research results. ${ }^{6}$

To treat tumors and inhibit their angiogenesis is one of the important approaches. Without adequate supply of diffuse blood, discharge of metabolic waste, and sufficient nutrients, tumor can only grow to a size of approximately $2 \mathrm{~mm}$. In the course of tumor formation, VEGF secreted by tumor endothelial cells and extra-cellular matrix, has an important influence on the formation of tumor vascular networks. VEGF is the most powerful mitogen of endothelial cells found so far and is a key regulator of stimulating tumor angiogenesis, vascular remodeling, and angiogenic sprouting. Therefore, it is of great significance to look for safe and effective vascular inhibitors, especially to develop natural herbal extracts with vascular inhibition. EGFR in vascular endothelial cells in lung cancer and other tumor tissues are highly expressed, and can accelerate the growth of tumor blood vessels by promoting VEGF expression.

The results of this study showed that compared with the model control group, the protein of VEGF and EGFR in 
the tumor tissue of the APS groups significantly decreased. The index of immune organs of spleen and thymus in the APS groups with high, middle and low dosages, respectively, was higher than that in model control group. It is suggested that APS may reduce the expression of VEGF and EGFR to improve the function of immune organs and thus inhibit tumors.

One limitation of this study is that positive control is not included, which in the future, can be further studied.

\section{REFERENCES}

1. Saito M, Shiraishi K, Goto A, Suzuki H, Kohno T, Kono K. Development of targeted therapy and immunotherapy for treatment of small cell lung cancer. Jpn J Clin Oncol 2018; 48:603-8.

2. Wu J, Wang J, Su Q, Ding W, Li T, Yu J, et al. Traditional Chinese medicine Astragalus polysaccharide enhanced antitumor effects of the angiogenesis inhibitor apatinib in pancreatic cancer cells on proliferation, invasiveness, and apoptosis. Onco Targets Ther 2018; 11:2685-98.

3. Reck M, Crinò L. Advances in anti-VEGF and anti-EGFR therapy for advanced non-small cell lung cancer. Lung Cancer 2009; 63:1-9.

4. Ge L, Shan Z, Han Q, Zhang N, Zhao Y. Effectiveness of allogeneic CD3AK cells on transplanted human renal cell cancer in mice with severe combined immune deficiency. J BUON 2016; 21:399-406.

5. Sun X, Xu Y, Wang Y, Chen Q, Liu L, Bao Y. Synergistic inhibition of thalidomide and icotinib on human non-small cell lung carcinomas through ERK and AKT signaling. Med Sci Monit 2018, 24:3193-203.

6. Zhuang M, Liu D, Chen Y, Ming H, Li Y. Inhibitory effect and the mechanism of astragalus polysaccharide combined with cisplatin on growth of inplanted Lewis lung carcinoma in mice. Chin J Cell Mol Immunol 2017; 33:503-7.

.......... 